

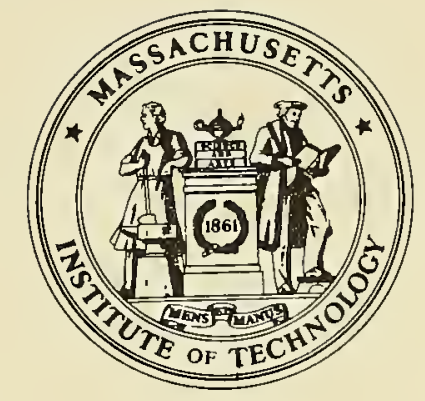

\section{LIBRARY}

OF THE

MASSACHUSETTS INSTITUTE OF TECHNOLOGY 


\section{Digitized by the Internet Archive in 2011 with funding from Boston Library Consortium Member Libraries}




\section{working paper department of economics}

\section{EQUITY, ENVY, AND EFFICIENCY * \\ by}

Hal R. Varian

Number 115

August 1973

\section{massachusetts instifute of
technology}

50 memorial drive cambridge, mass. 02139 


\section{EQUITY, ERYYY, AHD EFFICIENICY* \\ by}

Hal R. Varian

itumber 115

August 1973

"I wish to thank Daniel McFadden, Davic Gale, Guard Debreu and Carl Futia for helpful suggestions and comments. Of course, I an soleiy responsibie for any remaining errors. I wish also to thank the lational science Foundation for fellowship support during the writing of this paper. 


\title{
EQUITY, ENVY, AND EFFICIENCY
}

\author{
Hal R. Varian
}

\section{Contents :}

I. The Concept of Fairness 1

II. Fair Division 10

III. Fairness and Production 29

IV. Coalition Fairness 43 


\section{THE CONCEPT OF FAIRNESS}

In this section, I wish to examine the problem of fair division: given a fixed number of agents and a fixed amount of certain goods, what is a fair way to divide the goods among the agents?

I think that nearly everyone's impulse here is to say "evenly." Given the description of the above problem, that is perhaps the best we can do. However, if we allow ourselves further information about the tastes of the agents in question, we can achieve a more satisfactory solution. Thus if we bring preferences into consideration, we can see that even division is no longer satisfactory as an equity concept. For in general, if agents have different tastes an even distribution will not be pareto efficient and thus there will be incentive to trade. Now it is true that "everyone gains from trade", but some people may gain more than others. Thus in the trade from a pareto inefficient to a pareto efficient allocation, any equity inherent in the original situation may be destroyed. (See example 1.1) Since we are presumably interested not only in starting with a fair allocation, but also in keeping one, we will restrict ourselves to allocations that are stable with respect to potential for trade; that is, pareto efficient a1locations.

There is another basis for restricting ourselves to only pareto efficient allocations: if a given 
allocation is not pareto efficient, there will be an allocation where everyone can be made better off. If everyone can be made better off, we might as well do it, or so it would seem.

Since as far as we know the original situation in the division problem is symmetric - there are only the $\mathrm{n}$ agents and the bundle of $\mathrm{k}$ goods to be divided -. we would want to require that the final allocation be symmetric, that is, symmetric in preferences. What does this mean? We11, I would submit that an allocation would be symmetric in preferences if no agent preferred what any other agent had to what he had; that is, if there were no agents that envied other agents. Since the natural assumption in the fair division problem is that all agents have an equal prior claim to the original bundle, we would want the final allocation to reflect this initial symmetry, and any perceived asymmetry on the part of the agents can not be tolerated.

We are thus led to make the following formal definitions :

An allocation is equitable if and only if no agent prefers any other agent's bundle to his own. ${ }^{1}$ An allocation is fair if and only if it is both equitable and pareto efficient. ${ }^{2}$

Thus a fair allocation is doubly stable: There is no way to make any one agent better off without making some other agent worse off, and there is no agent who 
would rather have what some other agent has as opposed to what he has.

This is of course only a minimal requirement for fairness, based as it is only on facts concerning the physical allocation and the preferences of the agents. Thus we can easily construct cases where other considerations are of primary importance in determining whether a particular allocation is fair. Examples of such other considerations might be the strengths of the agents' preferences, the moral worth of the agents, or their contribution to the formation of the original bundle.

But the fact that we can construct such counterexamples should not detract from the interest of the original definition. For any ethical principle can admit of exceptions, precisely because any ethical principle can concern itself with only a fixed number of facts.

We can view the attempt to formulate ethical principles as an attempt to model ethical behavior in the same way that the formulation of grammatical principles serves to model linguistic behavior. In the ethical principles, as well as in the grammatical principles, there will be exceptional cases; this is true of any attempt to model behavior. But this is not a limitation of models as much as a defining feature; we want models because they are a simplification of reality, and as such they must admit of exceptions: a map on a one to one 
scale is completely useless.

As we enlarge our módel to bring in possibilities of production, possibilities of coalition formation, and so on, our criteria for what should count as a fair allocation may certainly change. However, we must walk before we can run, and we will first examine the implications of this simple definition in some detail.

Before I proceed to that task however, I wish to spend a small amount of time comparing this notion of fairness to two other ways the problem of fair division might be handled using other theories of normative economics; by doing so, I hope that the particular strengths and weaknesses of these theories can be clarified. The two other theories are those of classical welfare economics and of social decision theory.

Now both of these approaches, as well as that of fairness, agree that the question of whether an allocation is "good" depends, at least in part, on how the agents feel about that allocation. For purposes of clarifying these three approaches, we will decompose an agent's feelings into three components:

(1) how he feels about what he gets

(2) how he feels about what others get, independently of his own bundle

(3) how he feels about the differences between his own and others' bundles. We can examine the three theories with respect to 
which of these feelings they count as legitimate variables for the problem of determining whether a particular allocation is "good."

In classical welfare theory we are supposed to have a welfare function that depends on the agents' utility functions, which in turn are defined on each individual's bundle of goods. Thus the only type of feelings that are counted as relevant are those of type (1). It is true that distributional considerations may enter into consideration through the particular form of the social welfare function, but the form of the social welfare function is completely exogenous to the problem.

It is just this problem -- where does the social welfare function come from? - - that social decision theory was designed to answer. The form of the social welfare function should presumably depend on how agents feel about entire allocations, that is, on the feelings of types (1), (2) and (3). But by allowing preferences to be defined over entire allocations, social decision theory has allowed these feelings to enter into the problem in a completely uncontrolled way.

Now it may be true that it is a psychological fact that some agents do have preferences over entire allocations, but it is of some question whether all types of these "interpersonal" preferences should be counted as equally legitimate on ethical grounds. For example, suppose that I always want my neighbor to have Iess than 
I do; or that I want to ensure that no one be able to read Lady Chatterly's Lover; or that all people with black skins be required to use separate restroom facilities. Are these preferences to be counted with the same strength as "individualistic" preferences? To take an example somewhat closer to the spirit of the problem of fair division, consider a case where there are three agents, A, B, and $\mathrm{C}$, and consider a given allocation, $\mathrm{x}$. Suppose that A and B could both be made better off by trading while C's allocation would not change. Would the fact that C's utility would fall because of his jealousy of A be a relevant consideration to a social decision maker?

In the theory of fairness, we avoid these kinds of problems by allowing all three types of feelings to interact, but only in a constrained way. The three types of feelings mentioned before are constrained as follows:

(1) preferences are defined only on individual bundles

(2) one can judge another's bundle only on the same terms that he judges his own

(3) one can complain about another agent's bundle only if he would actually rather have his bundle instead of his own.

By forcing interpersonal comparisions to be made on the same basis as personal comparisons we have attempted to introduce some of the classical notions of symmetry 
in ethical principles; for example, "Do unto others as you would have them do unto you." Thus in the theory of fairness we allow "interpersonal" comparisions to be made, but only in a constrained and well. defined way.

It is clear that an important restriction of the theory of fairness -- which it shares with classical welfare theory - - is that utility functions be defined only on individual bundles. Notice however that the motivation is entirely different; classical welfare theory took this as a psychological given while the theory of fairness requires that this restriction be made on ethical grounds. 


\section{APPENDIX TO SECTION 1}

Example 1.1. An example of a trade to the efficient set (in fact, to the core) from an equal division endowment that does not preserve equity:

$$
\begin{aligned}
& u_{1}(x, y)=y \\
& u_{2}(x, y)=x \\
& u_{3}(x, y)=y
\end{aligned}
$$

initial bundle to be divided $=(3,3)$

final allocation Agent $1:(0,1.8)$

Agent 2: $(3,0)$

Agent 3: $(0,1.2)$

Here all agents have gained from trade, but agent 1 has gained much more than agent 3 . 
NOTES AND ACKNOWLEDGMENTS

1. The definition of equity is due to Foley [F].

2. The definition of fairness is due to Schmeidler and Yaari [SY].

\section{REFERENCES}

[F] Foley, D., "Rescource Allocation and the Public Sector," in Yale Economic Essays, Volume 7, Spring, 1967 .

[SY] Schmeidler, D., and Yaari, M., "Fair Allocations," unpublished. 


\section{FAIR DIVISION}

In this section I will present some theorems concerning the problem of fair division previously introduced and discuss some of the relationships between the concepts of equity, envy, and efficiency. We will first restate the previous definitions in somewhat more formal terms.

An allocation $x$ is weakly efficient ( $x$ is in PW) iff there is no feasible allocation $y$ such that $y_{i}>_{i} x_{i}$ for all agents $i$. An allocation $x$ is strongly efficient ( $x$ is in PS) iff there is no feasible allocation $y$ such that $y_{i} \succsim_{i} x_{i}$ for all agents $i$ and there is some agent $j$ such that $y_{j} \succ_{j} x_{j}$. An allocation $x$ is equitable iff $x_{i} \succsim_{i} x_{j}$ for all agents $i$ and $j$. If $x_{i} \prec_{i} x_{j}$ we will say that $i$ envies $j$ at the allocation $x$. If an allocation $x$ is both equitable and strongly efficient, we will say $x$ is fair. If the allocation $x$ is equitable but only weakly efficient, we will say $x$ is weakly fair.

A fundamental relationship between envy and efficiency is given in the following theorem.

THEOREM 2.1. If $x$ is a strongly efficient allocation, then there is some agent that envies no one and there is some agent that no one envies.

Thus there is a "top" and a "bottom" to the set of agents in a strongly efficient allocation. It is possible to extend this partial order to the whole set of 
agents by disregarding the non-envious agents and their bundles and considering the resulting allocation; this allocation is still strongly efficient, and thus there are non-envious agents. (These are the agents who envied only the original non-envious agents.) We can consider these agents to be the "second best off", and then continue to extend the ordering. Unfortunately simple examples show that the ordering which comes from disregarding the unenvied agents, those at the bottom of the pile, will not in general be consistent with the ordering just described. Nevertheless, it is of some interest to note that we can get a natural measure of how well off each agent is in any strongly efficient allocation.

Moving on to the concept of equity, we recall that a classical notion of equity in the context of a market economy is that of an equal income competitive equilibrium, which is also of course an efficient allocation. It is therefore reassuring to notice that equal income competitive allocations are indeed fair by our definition:

THEOREM 2.2. Suppose that preferences are monotonic.

Then if $(x, p)$ is a competitive equilibrium with $p \cdot x_{i}=p \cdot x_{j}$ for a 11 and $j$, then $x$ is fair. ${ }^{1}$

Interestingly enough, a competitive equilibrium from an equitable allocation is not necessarily fair, and not al1 fair allocations have equal incomes. (See examples 2.1 and 2.2.) 
A primary concern about the usefulness of the concept of fair allocations is the question of whether they exist in general circumstances. The above theorem gives us an immediate result on this existence question: THEOREM 2.3. If preferences are convex and monotonic, then fair allocations exist. ${ }^{2}$

The primary restriction of the above theorem is that of convexity of preferences. As fair allocations can easily exist in the absence of this condition, the above result is somewhat unsatisfactory. (See examples 2.4 and 2.6.$)$

It turns out that a more general condition for the existence of fair allocations is that the topological structure of the set of efficient allocations be especially simple; that is, that it consist of one piece with no "holes" in it. The next theorem investigates conditions under which this is the case; here $\mathrm{PW}_{+}=\left\{x\right.$ in $\mathrm{PW}: \mathrm{x}_{i} \neq 0$ for any $\left.i\right\}, u\left(\mathrm{PW}_{+}\right)=\left\{\left(\mathrm{u}_{1}\left(\mathrm{x}_{1}\right) \ldots\right.\right.$ $\left.u_{n}\left(x_{n}\right)\right):\left(x_{1} \ldots x_{n}\right)$ is in $\left.\mathrm{PW}_{+}\right\}$.

THECREM 2.4. Suppose that every agent prefers any non-zero bundle to the zero bundle; then $u\left(\mathrm{PW}_{+}\right)$is homeomorphic to the interior of an n-1 dimensional simplex. Furthermore, if there are no two allocations in $\mathrm{PW}_{+}$which all agents regard as indifferent, then $\mathrm{PW}_{+}$ is itself homeomorphic to the interior of an n-1 dimensional simplex. ${ }^{3}$ 
COROLLARY If preferences are monotonic and strictly convex, then PW = PS and both are homeomorphic to an n-1 dimensional simplex. 4

The assumptions that underly the above theorem, when coupled with the results of Theorem 2.1 , are enough to give the existence of fair allocations.

THEOREM 2.5 If preferences are monotonic and there are no two allocations in $P W$ which all agents regard as indifferent, then fair allocations exist. ${ }^{5}$

The restrictive assumption in the above theorem is clearly the assumption about the two "pareto indifferent" efficient allocations. This is of course precluded by strict convexity of preferences, but as example 2.3 shows, this condition cannot be dispensed with if we want the efficient set to be homeomorphic to a simplex, and the existence of fair allocations. However we can weaken the notion of fairness somewhat and find a more general concept (though ethically less satisfactory) that will exist without the above condition. Consider the fair division problem as a bargaining process. Given a strongly efficient allocation $x$ we will say that agent $i$ objects to agent $j$ if: (1) i envies $j$; and (2) i can propose a new allocation $y$ that all agents regard as indifferent to $x$ where no one envies $j$. An objection to this new allocation $y$ by any other agent can be called a counterobjection. Then we can define the fair set of allocations, $F$, as being the set of 
strongly efficient allocations where every objection has a counterobjection. Thus in the fair set there are either no envies, or the envies tend to "cancel out." We can show that:

THEOREM 2.6. If preferences are monotonic then fair sets exist.

A fundamental fact of our ethical notions is that "equals should be treated equally." In the context of the fair division problem, we can interpret "equals" as agents who have exactly the same preferences. Then it is obvious from the definition of fairness that at a fair allocation "equals are treated equally" in the sense that they are indifferent to each other's bundles. Further results in this direction are:

THEOREM 2.7. Suppose the preferences of agent $i$ are identical with those of $j$ and both are strictly convex; then if $x$ is a fair allocation, $x_{i}=x_{j}$.

In particular, if all agents have identical strictly convex preferences, then equal division is the unique fair allocation. However, we can relax this condition of strict convexity to get a rather general result on existence in the special case of equal tastes: THEOREM 2.8. If any bundle is preferred to the 0 bundle and all agents have identical preferences, then weakly fair allocations exist.

(See example 2.5 which shows that this is not true for fair allocations.) 


\section{APPENDIX TO SECTION 2}

The set of feasible allocations will be denoted by $X=\left\{x\right.$ in $R_{+}^{n k}:\left\{x_{i} \leqq w\right\}$. We assume $w>0$. We will assume that each agent $i$ has preferences $\succsim_{i}$ defined on the commodity space $\mathrm{R}_{+}^{\mathrm{k}}$ and that these preferences are complete, transitive and closed. Thus these preferences can be represented by continuous utility functions $\mathrm{u}_{\mathrm{i}}: \mathrm{R}_{+}^{\mathrm{k}} \rightarrow \mathrm{R}$.

Preferences are said to be convex iff for $x^{\prime} \neq x$, $x^{\prime} \succsim_{i} x$ implies $a x+(1-a) x^{\prime} z_{i} x$ for $0 \leqq a \leqq 1$. Preferences are strictly convex iff, in the above case, $a x+(1-a) x^{\prime}>_{i} x$ for $0<a<1$. Preferences are monotonic iff $x \leqq x^{\prime}$ and $x \neq x^{\prime}$ implies $x \prec_{i} x^{\prime}$.

An allocation $x$ is a competitive equilibrium with prices $p$ and initial endowment $\omega$ iff $x^{\prime} \succ_{i} x_{i}$ implies that $\mathrm{p} \cdot \mathrm{x}^{\prime}>\mathrm{p} \cdot \mathrm{x}_{i}$ and $\mathrm{p} \cdot \mathrm{x}_{i} \leqq \mathrm{p} \cdot \omega_{i}$ for $\mathrm{i}=1, \ldots, \mathrm{n}$.

$$
\mathrm{S}^{\mathrm{n}-1}=\left\{\mathrm{x} \text { in } \mathrm{R}_{+}^{\mathrm{n}}:\left[\mathrm{x}_{i}=1\right\}=\text { the unit } \operatorname{simplex}\right.
$$

int $S^{n-1}=\left\{x\right.$ in $S^{n-1}: x_{i}>0$ for all $\left.i=1, \ldots n\right\}$

$$
S_{j}^{n-1}=\left\{x \text { in } S^{n-1}: x_{j}=0\right\}=\text { the } j^{\text {th }} \text { side of } S^{n-1}
$$

THEOREM 2.1. If $x$ is a strongly efficient allocation, then there is some agent that envies no one and there is some agent that no one envies.

Proof. Suppose to the contrary that each agent envies some other agent. Then, since there are only a finite number of agents, there is some cycle $\left(i_{1} \ldots i_{m}\right)$ such that 
$i_{1}$ envies $i_{2}$ envies...envies $i_{m}$ envies $i_{1}$. Then the allocation $x^{\prime}$ where each agent in the cycle receives the bundle of the agent he envies and agents outside the cycle remain the same is feasible and dominates the original allocation $x$. This contradicts the fact that $x$ is strongly efficient.

The proof of the second assertion is similar. $\square$

THEOREM 2.2. Suppose that preferences are monotonic.

Then if $(x, p)$ is a competitive equilibrium with $p \cdot x_{i}=p \cdot x_{j}$ for al1 $i$ and $j, x$ is fair. ${ }^{1}$

Proof. First we will show that $x$ is strongly efficient. Assume not; then there is some allocation $y$ such that $y_{i} \succsim_{i} x_{i}$ for $i=1, \ldots, n$ and for some $j, y_{j} \succ_{j} x_{j}$. We can choose $y$ so that it itself is strongly efficient.

For each $j$ that strictly prefers $y_{j}$ to $x_{j}$ we have $p \cdot y_{j}>p \cdot x_{j}$. Consider some agent $i$ that is indifferent between $x_{i}$ and $y_{i}$, if any such agents exist. If $p \cdot y_{i}<p \cdot x_{i}$ the agent could afford to buy a slightly more expensive bundle, and by monotonicity he could find a bundle strictly better than $x_{i}$, contradicting the fact that $x_{i}$ is a competitive equilibrium. Thus

$$
p \cdot y_{i} \geqq p \cdot x_{i}
$$

so that

$$
\sum \mathrm{p} \cdot \mathrm{y}_{\mathbf{i}}>\sum \mathrm{p} \cdot \mathrm{x}_{\mathbf{i}}
$$

Since preferences are monotonic,

$$
\sum y_{i}=\sum x_{i}=\sum \omega_{i}
$$


but this gives us

$$
\mathrm{p} \cdot \sum \omega_{i}>\mathrm{p} \cdot \sum \omega_{i} .
$$

This is a contradiction, so it must be that $x$ is strongly efficient.

To show that $x$ is also equitable, we suppose that agent $i$ envies agent $j: x_{i} \prec_{i} x_{j}$. Then by definition of competitive equilibrium, $\mathrm{p} \cdot \mathrm{x}_{i}<\mathrm{p} \cdot \mathrm{x}_{j}=\mathrm{p} \cdot \mathrm{x}_{i}$ which is a contradiction.

THEOREM 2.3. If preferences are convex and monotonic, then fair allocations exist. ${ }^{2}$

Proof. Let the initial allocation $\omega$ be defined by $\omega_{i}=w / n$. Under the assumptions of the theorem, standard existence proofs show that a competitive equilibrium $(x, p)$ will exist, be in PS, and $p \cdot x_{i}=p \cdot x_{j}=p \cdot(w / n)$. By Theorem 2.2 this will be fair.

THEOREM 2.4. Suppose that every agent prefers any nonzero bundle to the zero bundle; then $u\left(\mathrm{PW}_{+}\right)$is homeomorphic to the interior of an $\mathrm{n}-1$ dimensional simplex. Furthermore, if there are no two allocations in $\mathrm{PW}_{+}$ which all agents regard as indifferent, then $\mathrm{PW}_{+}$is itself homeomorphic to the interior of an $n-1$ dimensional simplex. ${ }^{3}$

Proof. We will without loss of generality normalize the utility functions so that $u_{i}(0)=0$ and $u_{i}(w / n)=1$. The proof procedes in a number of steps: 
Step 1: If feasible $x \gg 0$ is not in $\mathrm{PW}$, then there exists a feasible allocation $z$ and a real number $t>1$ such that $u_{i}\left(z_{i}\right)=i t u_{i}\left(x_{i}\right)$ for all $i=1, \ldots, n$.

Proof. If $x$ is not in $P W$, then there exists some feasible y such that $u_{i}\left(y_{i}\right) / u_{i}\left(x_{i}\right)>1$ for $i=1, \ldots, n$. Let $t=\min _{i} u_{i}\left(y_{i}\right) / u_{i}\left(x_{i}\right)$. The functions $f_{i}:[0,1] \rightarrow R$ defined by $f(e)=u_{i}(e y) / u_{i}(x)$ are continuous, $f_{i}(1) \geqq t, f_{i}(0)=0<t$. Therefore, by the intermediate value theorem, there is a set of $e_{i}^{\prime}$ such that $f_{i}\left(e_{i}^{\prime}\right)=t$; the allocation defined by $z_{i}=e_{i}^{\prime} y_{i}$ is feasible and satisfies the above requirements.

Step 2: Let $\mathrm{p}: \mathrm{u}\left(\mathrm{PW}_{+}\right) \rightarrow$ int $\mathrm{S}^{\mathrm{n}-1}$ be defined by $p(u)=u /\left[u_{i}\right.$. The function $p$ is certainly continuous on $\mathrm{u}\left(\mathrm{PW}_{+}\right)$since the denominator cannot vanish. Furthermore I claim it is one to one on $u\left(\mathrm{PW}_{+}\right)$.

Proof. Assume not; then there exists $u, v$ in $u\left(P W_{+}\right)$such that $u /\left(\sum u_{i}\right)=v /\left(\sum v_{i}\right)=z$ in int $s^{n-1}$. Therefore the $u$ and $v$ are scalar multiples of each other with no zero co-ordinates so that one is strictly greater than the other, which contradicts the fact that both are in $\mathrm{u}\left(\mathrm{PW}_{+}\right)$.

Step 3: The set $T(y)=\{t$ in $R:$ ty is in $u(x)\}$ contains a nonzero element.

Proof. The set of functions $g_{i}:[0,1] \rightarrow R$ defined by $g_{i}\left(e_{i}\right)=u_{i}\left(e_{i}(w / n)\right)$ are continuous, $g_{i}(1)=1$, $g_{i}(0)=0$. Applying the intermediate value theorem again we find that for $y$ in $s^{n-1}$ there exists $e_{i}^{\prime}$ in $[0,1]$ 
such that $u_{i}\left(e_{i}^{\prime}(w / n)\right)=y_{i}$ and the allocation $e_{i}^{\prime}(w / n)$ for $i=1, \ldots, n$ is certainly feasible. Therefore 1 is in $\mathrm{T}(\mathrm{y})$.

Step 4: The function $\mathrm{p}: \mathrm{u}\left(\mathrm{PW}_{+}\right) \rightarrow$ int $\mathrm{S}^{\mathrm{n}-1}$ is onto. Proof. Given $y$ in int $S^{n-1}$ the set $T(y)$ defined above is compact and nonempty. Then there exists $0 \neq t^{\prime}=$ the maximum $t$ such that $t$ is in $T(y)$. Suppose that t'y is not in $u\left(\mathrm{PW}_{+}\right)$; then by step 1 there is some $t^{\prime \prime}$ such that $t^{\prime \prime} t^{\prime} y$ is in $u(X)$. But then $t^{\prime \prime} t t^{\prime}>t^{\prime}$ which contradicts the maximality of $t^{\prime}$.

Step 5: $\mathrm{p}^{-1}$ is continuous on int $\mathrm{s}^{\mathrm{n}-1}$.

Proof. Let $\mathrm{K}$ by an arbitrary closed set in $\mathrm{u}\left(\mathrm{PW}_{+}\right)$. Then $K$ is compact since it is a subset of a compact set, namely $u(P W)$. For $\mathrm{p}^{-1}$ to be continuous we need $\left(\mathrm{p}^{-1}\right)^{-1}(\mathrm{~K})$ to be closed. Since $\mathrm{p}$ is one to one and onto, this set is just $p(K)$ which is closed by compactness of $K$.

Step 6: $p$ is a homeomorphism between $u\left(\mathrm{PW}_{+}\right)$and int $S^{n-1}$.

Proof. It is one to one, continuous, and onto. Furthermore, if there are no two allocations $x$ and $y$ in $\mathrm{PW}_{+}$such that $\mathrm{u}_{i}\left(\mathrm{x}_{i}\right)=u_{i}\left(\mathrm{y}_{i}\right)$ for $i=1, \ldots, \mathrm{n}$ the map $u$ restricted to $\mathrm{PW}_{+}$will be one to one. It is clearly continuous and onto, so composing it with $\mathrm{p}$ will give us a homeomorphism between $\mathrm{PW}_{+}$and int $\mathrm{s}^{\mathrm{n}-1} \cdot \square$

COROLLARY. If preferences are strongly monotonic and 
strictly convex, then PS $=$ PW and both are homeomorphic to an $\mathrm{n}-1$ dimensional simplex. ${ }^{4}$

Proof. That PW contains PS is obvious. We will show that PS contains PW. Suppose the allocation $x$ is not in PS; then there is some allocation $y$ such that $u_{i}\left(y_{i}\right) \geqq u_{i}\left(x_{i}\right)$ for all $i$ and $u_{j}\left(y_{j}\right)>u_{j}\left(x_{j}\right)$ for some $j$. By continuity we can remove a positive fraction (1- $\theta$ ) of all commodities from $y_{j}$ and still have $u_{j}\left(\theta y_{j}\right)>$ $u_{j}\left(x_{j}\right)$. Then define an allocation $z$ by: $z_{j}=y_{j}$, $z_{i}=y_{i}+(1-\theta) y_{j} /(n-1)$ for $i=1, \ldots, n, i \neq j$. The allocation $z$ has the property that $u_{i}\left(z_{i}\right)>u_{i}\left(x_{i}\right)$ for all $i=1, \ldots, n$ so that $x$ is not in $\mathrm{PW}$.

We now need to show that there are no two allocations in $\mathrm{PW}$ which all agents regard as indifferent. Suppose that $\mathrm{x}$ and $\mathrm{y}$ are two such allocations; then $(1 / 2) \mathrm{x}+$ $(1 / 2) y$ is feasible, at least as good for all agents, and strictly preferred to both $\mathrm{x}$ and $\mathrm{y}$ by agents for whom $x_{i} \neq y_{i}$, contradicting the efficiency of $x$ and $y$. The fact that the homeomorphism $p$ is one to one and onto on the boundary of PW can be verified from the fact that $\mathrm{PS}=\mathrm{PW}$ and the steps of the theorem.

THEOREM 2.5. If preferences are monotonic and there are no two allocations in $\mathrm{PW}$ which all agents regard as indifferent, then fair allocations exist. ${ }^{5}$

Proof. By the remarks in Step 6 of Theorem 2.4 we see that $\mathrm{u}$ is a homeomorphism between $\mathrm{PW}_{+}$and $\mathrm{u}\left(\mathrm{PW}_{+}\right)$. In 
Theorem 2.6 we see that the intersection of the $u\left(M_{j}\right)$ 's is nonempty, and thus the intersection of the $M_{j}$ 's is nonempty. Any allocation in this intersection is fair.

LEMMA. (Knaster, Kuratowski, and Mazurkiewicz) Let $M_{1} \ldots M_{n}$ be a family of closed subsets of $S^{n-1}$ with the property that the $j^{\text {th }}$ face of $S^{n-1}$ is contained in $M_{j}$, and that $S^{n-1}$ is contained in the union of the $M_{j}$. Then the intersection of the $M_{j}$ is nonempty.

Proof. See Graves, [G], pages 146-148.

THEOREM 2.6. If preferences are monotonic then fair sets exist.

Proof. Define the set of allocations where no agent envies agent $j: M_{j}=\left\{x\right.$ in PS: $u_{i}\left(x_{i}\right) \geqq u_{i}\left(x_{j}\right)$ for all $i=1, \ldots, n\}$. Then the union of these sets for $\mathrm{j}=1, \ldots, \mathrm{n}$ covers PS by Theorem 2.1 , and by the Corollary to Theorem 2.4, PW = PS. Since the functions $u_{i}$ are continuous $M_{j}$ is closed, and since any bundle is preferred to the zero bundle, $\mathrm{M}_{j}$ contains all allocations in PS where $x_{j}=0$.

Now take the image of these sets $M_{j}$ under the map pu: PS $\rightarrow S^{n-1}$ defined by pu(x) $=\left(u_{1}\left(x_{1}\right) \ldots u_{n}\left(x_{n}\right)\right) /$ $\left\{u_{i}\left(x_{i}\right)\right)$. The sets $p u\left(M_{j}\right) j=1, \ldots, n$ satisfy the hypotheses of the lemma of Knaster, Kuratowski, and Mazurkiewicz, so that their intersection is nonempty. Let $z$ by in this intersection; then $z>0$ since 
if $z_{i}=0$ for some $i$, ther'e would be some $j$ such that $z_{j}>0$ and therefore $p^{-1}(z)$ would not be in $u\left(M_{j}\right)$. Since the points where $z \gg 0$ form the interior of $s^{n-1}$, $p$ is a homeomorphism on such points so that the intersection of the $u\left(M_{j}\right)$ is non-empty for $j=1, \ldots, n$. Let $v$ be in this intersection and consider the set $u^{-1}(v)$, which I claim is a fair set, F.

This is true because:

(1) $u_{i}\left(x_{i}\right)=u_{i}\left(y_{i}\right)$ for al1 $x, y$ in $F$, $i=1, \ldots n$.

(2) Suppose that $u_{i}\left(x_{j}\right)>u_{i}\left(x_{i}\right)$, so that $x$ is not in $M_{j}$ for some $x$ in $F=u^{-1}(v)$. But $v$ is in $u\left(M_{j}\right)$ so there must be some other allocation $y$ in $u^{-1}(v)$ that is in $M_{j}$; which means $u_{i}\left(y_{i}\right) \geqq u_{i}\left(y_{j}\right)$ for $i=1, \ldots n$ and $u_{i}\left(y_{i}\right)=u_{i}\left(x_{i}\right)$ for $i=1, \ldots, n$.

THEOREM 2.7. If the preferences of agent $i$ are identical with those of $j$ and both are strictly convex, then if $x$ is a fair allocation, $x_{i}=x_{j}$.

Proof. Assume that $x_{i} \neq x_{j}$, and consider the allocation $z=(1 / 2) x_{i}+(1 / 2) x_{j}$. Since $x$ is fair and $i$ and $j$ have the same preferences, $x_{i} \sim_{i} x_{j}$ and $x_{j} \sim_{j} x_{i}$ so that $z \succ_{i} x_{i}$ and $z \succ_{j} x_{j}$. Since giving $z$ to both $i$ and $j$ is feasible, this contradicts strong efficiency. $\square$ 
THEOREM 2.8. If any bundle is preferred to the 0 bundle and al1 agents have identical preferences, then weakly fair allocations exist.

Proof. If an allocation is to be fair in these circumstances, it must give equal utility to each agent and also be weakly efficient. Let pu be the map defined in Theorem 2.4 Step 2 ; then $(\mathrm{pu})^{-1}(1 / \mathrm{n} \ldots 1 / \mathrm{n})$ is a set of weakly efficient allocations with equal utilities. $\square$ 
Example 2.1. The allocation $x=\left(x_{1}, x_{2}\right)$ is equitable because it is preferred to its $\operatorname{swap} x^{\prime}=\left(x_{2}, x_{1}\right)$. The allocation $y$ is a competitive equilibrium from $x$, but $y$ is not equitable.

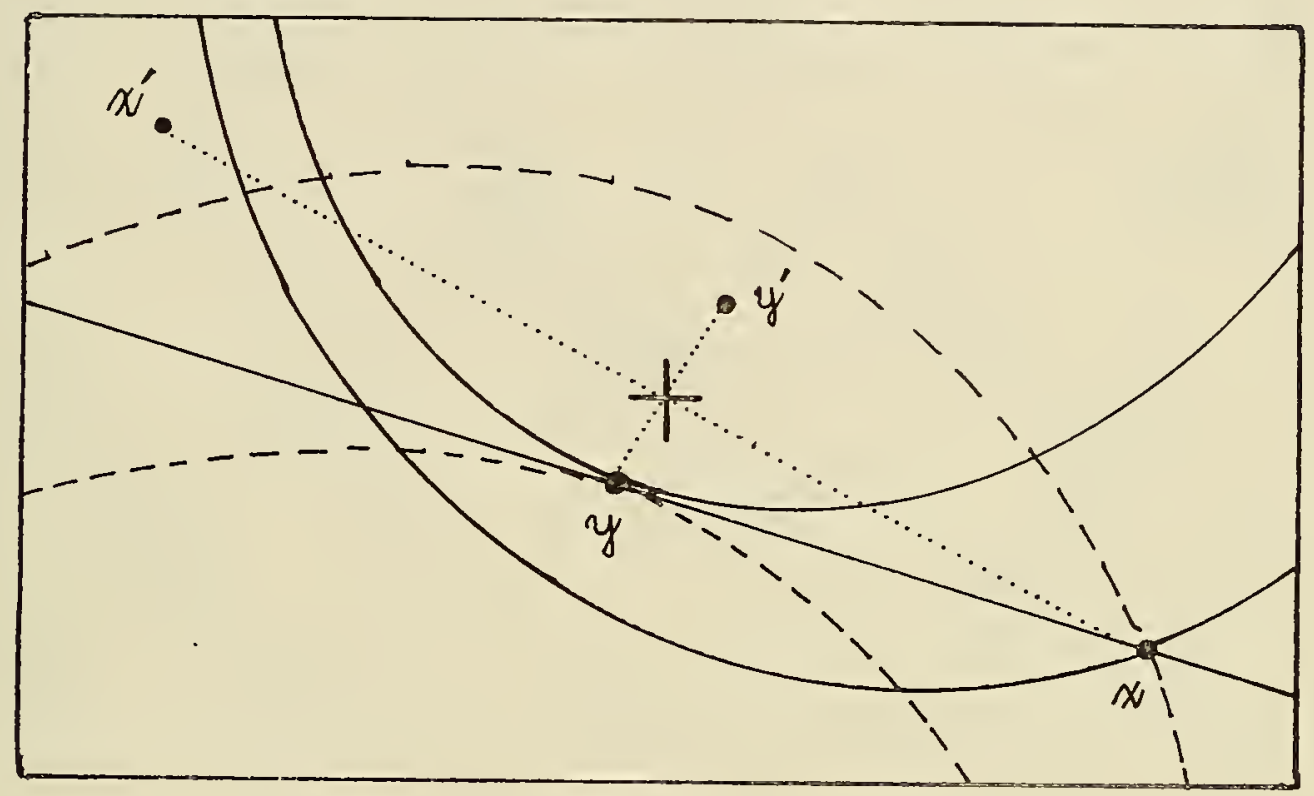

Example 2.2. The allocation $x$ is fair, but $x$ is not a competitive equilibrium with equal incomes, or even in the equal division core.

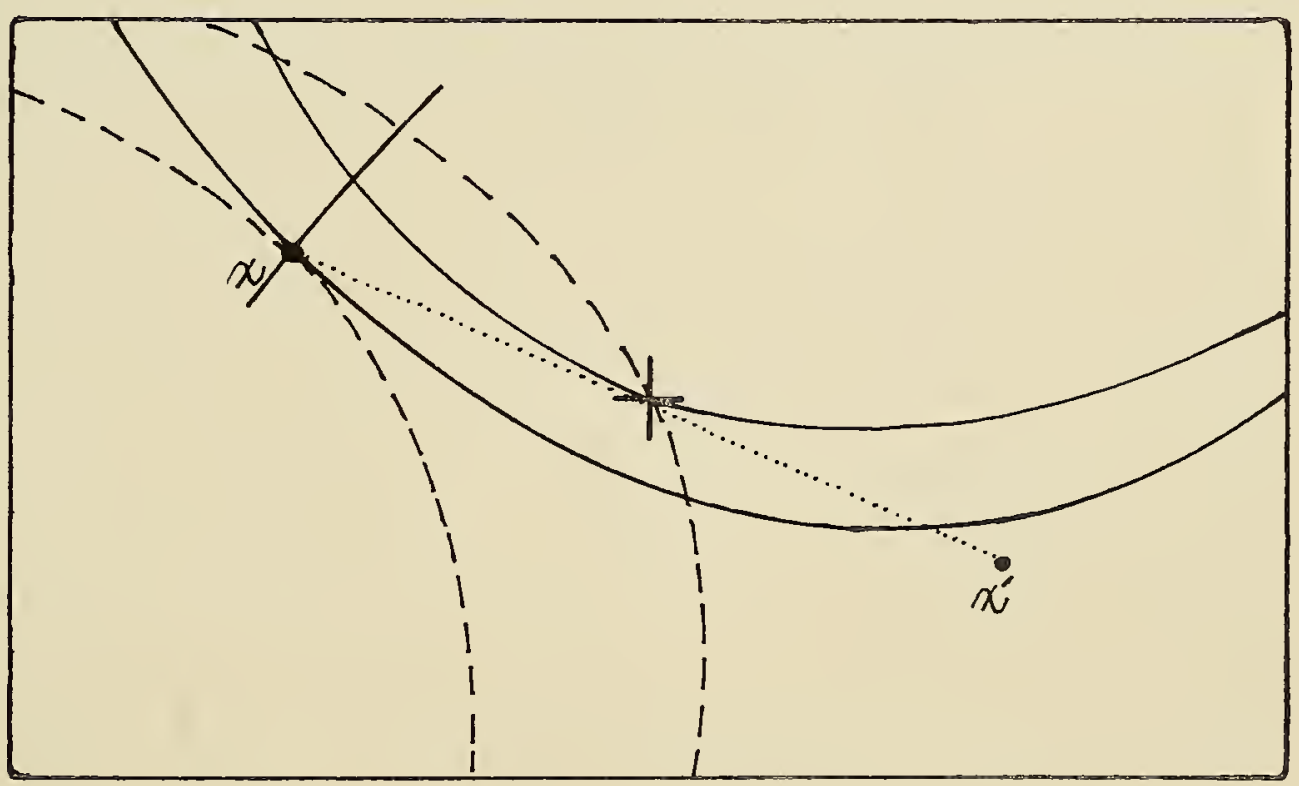


Example 2.3. Monotonicity does not imply the existence of fair allocations. (However, the allocations $x$ and $y$ form a fair set.)

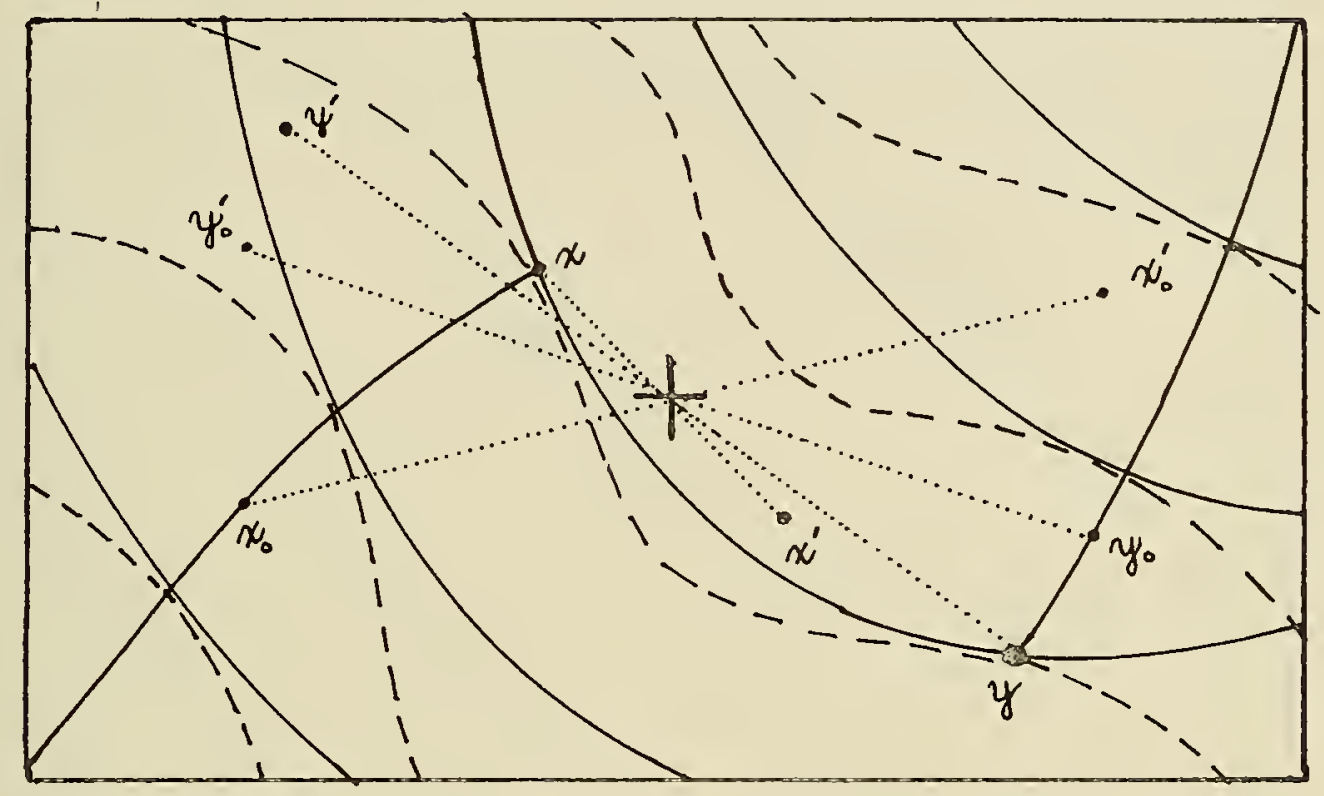

To see that there are no fair allocations in the diagram, imagine a point such as $x_{0}$ or $y_{0}$ that moves along one of the components of PS. As $x_{0}$ moves along the left component, its swap $x_{0}^{1}$ always lies on a higher indifference curve than does $x_{0}$, showing that $x_{0}$ cannot be fair. The point $y_{0}$ behaves similarly. 
Example 2.4. Convexity is not necessary for the existence of fair allocations.

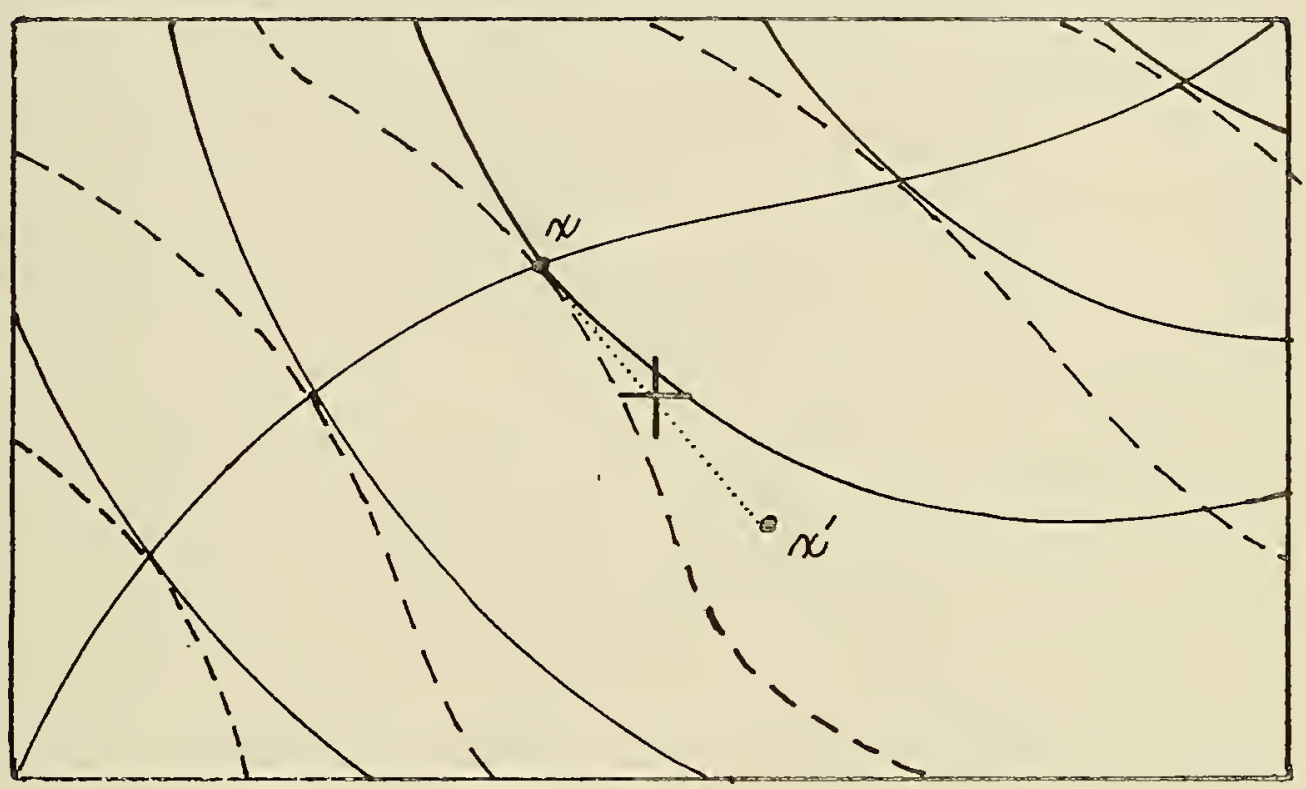

Example 2.5. Here $u_{1}\left(z^{1}, z^{2}\right)=u_{2}\left(z^{1}, z^{2}\right)=\max \left(z^{1}, z^{2}\right)$ and $w=(2,1)$. Only agent 1 has his indifference curves shown. PS consists of only the north-west and south-east corners; $\mathrm{PW}$ is drawn in heavy lines; $\mathrm{x}$ and $\mathrm{y}$ are weakly fair.

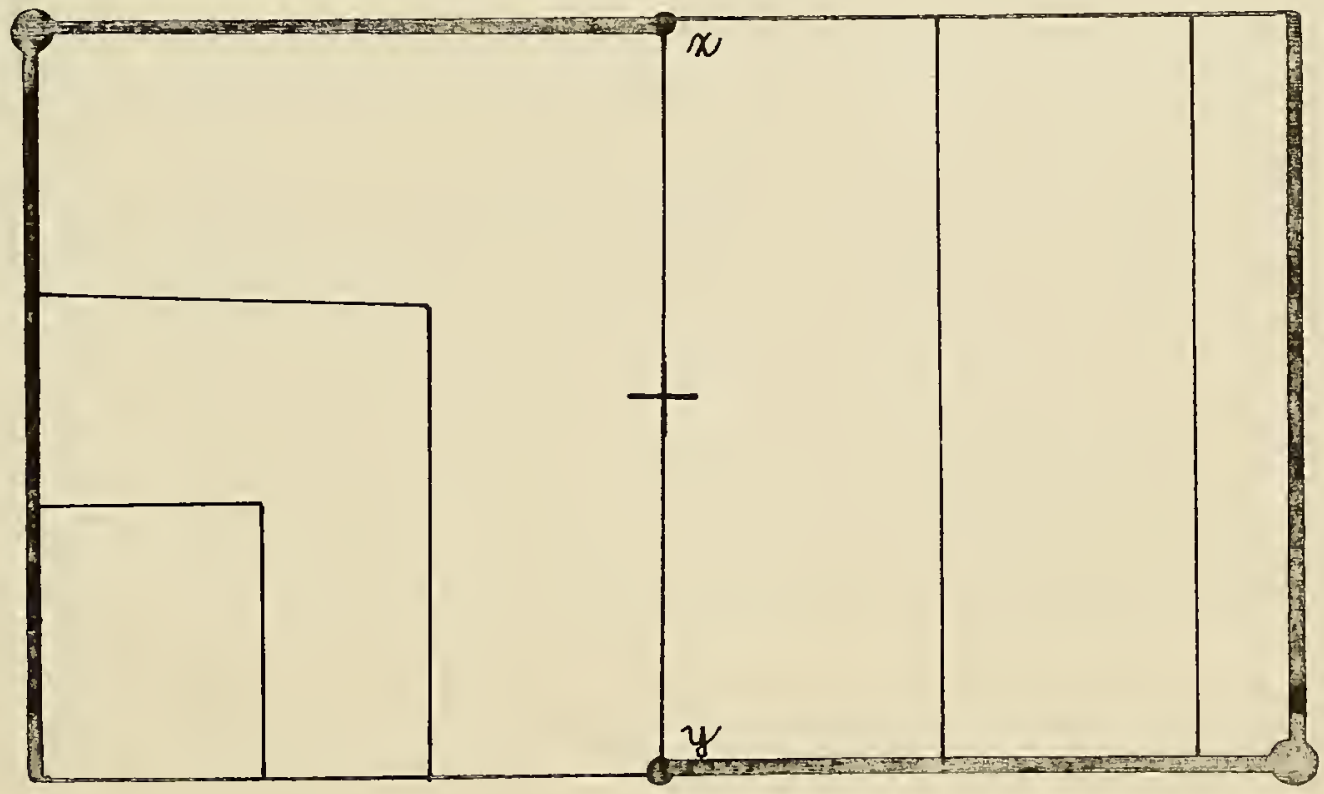


Example 2.6. The allocation $x$ is fair but a competitive equilibrium from $w / 2$ does not exist because of nonconvexities.

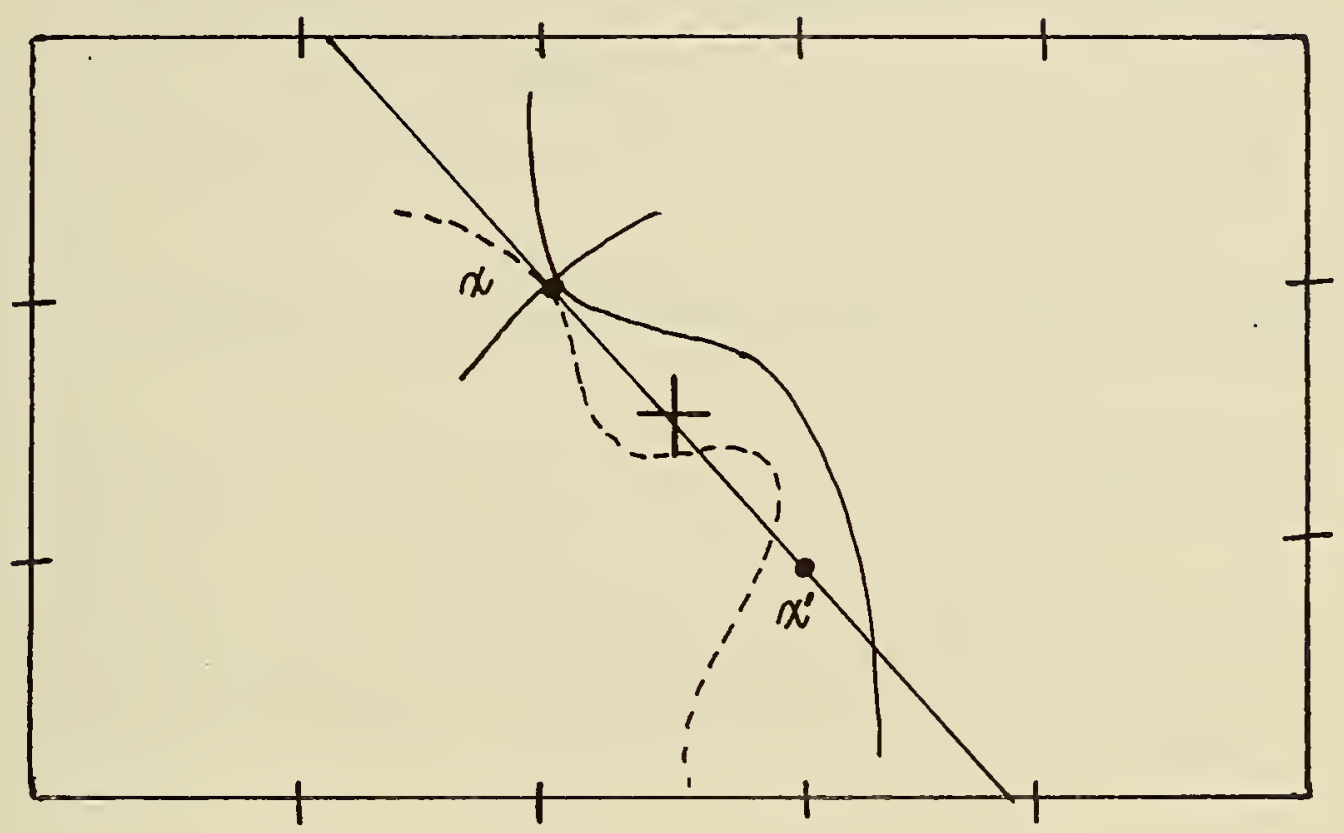


NOTES AND ACKNOWLEDGEMENTS

1. The first part of Theorem 2.2 is of course Koopmans' first optimality theorem. Since the definitions are slightly different, I have repeated the proof, inserting the necessary changes. The assumptions can be relaxed somewhat.

2. The idea of Theorem 2.3 is due to Schmeidler and Yaari [SY] .

3. The first part of Theorem 2.4 is a generalization of a lemma due to Arrow and Hahn [AH], page 111, which had required the use of convexity.

4. The corollary to Theorem 2.4 is not new although I have been unable to discover the exact reference.

5. The idea of using the Knaster-KuratowskiMazurkiewicz lemma here is due to Schmeidler and Yaari [SY]. The hypothesis of monotonicity can be replaced by the hypotheses that PS $=$ PW and any non-zero bundle is preferred to the zero bundle.

\section{REFERENCES}

[AH] Arrow, K.J., and Hahn, F.J., General Competitive Analysis, Holden-Day, Inc., SanFrancisco, 1971.

[G] Graves, L.M., The Theory of Functions of a Real Variable, New York, 1964 .

[SY] Schmeidler, D., and Yarri, M., "Fair Allocations," unpublished. 


\section{FAIRNESS AND PRODUCTION}

In Section 2 an important characteristic of the problem of fair division was that the bundle of goods to be divided was fixed, that is, there were no possibilities for production. Since the social resources were regarded as fixed, it is not surprising that we were able to discover an appealing definition of fairness and a relatively general theorem of existence. When the resources are fixed and no information but preferences is available, it is only natural to assume that everyone has an equal prior claim to the social resources. Thus any perceived asymmetry by the agents, such as envy, cannot be tolerated.

The situation is radically different when production is possible. For in this case the agents may contribute differently to the social product, and thus there is an inherent asymmetry to the problem. Indeed, the deepest problems of distributive justice are concerned precisely with this question: how do we divide the social product when agents can contribute differently to the formation of that product? We will try to investigate this question by extending the approach of fair division discussed in the last section.

We will assume that there is a fixed initial bundle of consumption goods $\mathrm{w}$ in $\mathrm{R}_{+}^{\mathrm{k}}$; furthermore, each agent can hold up to 1 unit of his own leisure. Thus the final bundles held by the $i^{\text {th }}$ agent are of the form $\left(x_{i}, 1-q_{i}\right)$ 
where $x_{i}$ is the $i^{\text {th }}$ agent's bundle of commodities, $q_{i}$ is his amount of labor time, and thus $1-q_{i}$ is his amount of leisure time. We will incorporate the technological production possibilities into the analysis by considering the set of all feasible allocations $x$, a subset of $R_{+}^{n(n+k)}$. The definition of strongly efficient allocations is similar to the previous definition, an allocation is equitable iff $\left(x_{i}, 1-q_{i}\right) \succsim_{i}\left(x_{j}, 1-q_{j}\right)$ for all agents $i$ and $j$, and an allocation is fair iff it is both equitable and strongly efficient. Thus if we have an efficient allocation where each agent prefers his consumption-leisure bundle to any other agent's, that allocation is fair.

The problem with this approach is simply this: fair allocations, as defined above, will not in general exist, even in very regular cases. The problem becomes apparent when we examine the proof of Theorem 2.6; for this theorem we need the results of both Theorem 2.4 (that the efficient set is homeomorphic to a simplex) and Theorem 2.1 (that at an efficient allocation there is some agent that no one envies.) There is no problem with Theorem 2.6; the efficient set will still be homeomorphic to a simplex if we assume that: (1) 0 consumption and 0 leisure is the worst possible bundle; and (2) the set of feasible allocations is regular; i.e., it is compact and convex, and if $(x, 1-q)$ is in $x$, every allocation that is smaller than $(x, 1-q)$ is in $x$. 
The problem comes in Theorem 2.1. Surprising as it may seem, it is possible to have strongly efficient allocations where two agents each envy the other. Consider the following two person, two good example:

$$
\begin{aligned}
& u_{1}\left(x_{1}, q_{1}\right)=\log x_{1}+\log \left(35-q_{1}\right) \\
& u_{2}\left(x_{2}, q_{2}\right)=\log x_{2}+\log \left(25-q_{2}\right) \\
& x_{1}+x_{2}=(1 / 5) q_{1}+q_{2}
\end{aligned}
$$

Consider the allocation $((6,5),(10,15))$. It is easy to check that the marginal rates of substitution equal the marginal rates of transformation so that this allocation is efficient. However

$$
\begin{aligned}
& u_{1}\left(x_{1}, q_{1}\right)=6 \times 30=180 \\
& u_{1}\left(x_{2}, q_{2}\right)=10 \times 20=200 \\
& u_{2}\left(x_{2}, q_{2}\right)=10 \times 10=100 \\
& u_{2}\left(x_{1}, q_{1}\right)=6 \times 20=120
\end{aligned}
$$

so that each agent envies the other. Since the crucial relationship between envy and efficiency does not go through to the production case, the proof of Theorem 2.6 does not work. In fact the following economy ${ }^{1}$ has no fair allocations at all, even though it exhibits constant returns to scale and homogeneous utility functions:

$$
\begin{aligned}
u_{1}\left(x_{1}, q_{1}\right) & =(11 / 10) x_{1}+\left(1-q_{1}\right) \\
u_{2}\left(x_{2}, q_{2}\right) & = \\
x_{1}+x_{2} & =(1 / 10) q_{2}+q_{1} \\
0 & \leqq q_{1} \leqq 1 \\
0 & \leqq q_{2} \leqq 1
\end{aligned}
$$


(For a proof, see the appendix to this section.)

It is important to notice that this non-existence is not due solely to the fact that there are different types of labor or different abilities. The effect of different tastes is crucial, for one can show that Theorem 2.8 goes through unchanged so that if all agents have the same preferences, a fair allocation exists, even though agents' abilities may differ.

Apparently to get a satisfactory notion of fairness in the production context, we will have to change our definition of "equity." We will consider two possible generalizations of the notion of fair allocations, each concentrating on different aspects of the concept of equity.

We will consider production technologies where it makes sense to associate with each agent the amount of goods that that agent produces at a given allocation. Thus we can consider bundles of the form $\left(x_{i}, 1-q_{i}, z_{i}\right)$ where $x$ and $q$ are as before and $z_{i}$ is the amount of all commodities produced by the $i^{\text {th }}$ agent, so that $z_{i}$ is an element of $\mathrm{R}^{\mathrm{k}}$. In what follows we will make the Independence Assumption: that $z_{i}$ is independent of permutations of the other $z_{j}{ }^{\prime} s$. Thus the production of the $i^{\text {th }}$ agent may depend on what others produce, but not on who produces it.

Under this assumption, it makes sense to ask how much $j$ would have to work to produce what $i$ produces at 
some particular allocation $(x, 1-q, z)$; this will just be the amount of j's labor necessary to produce the output $z_{i}$ assuming $z-z_{i}$ is held constant, and we will denote this amount of labor by $Q_{j}\left(z_{i}\right)$.

We shall now define an allocation as equitable* iff $\left(x_{i}, 1-q_{i}\right) \succsim_{i}\left(x_{j}, 1-Q_{i}\left(z_{j}\right)\right)$ for all agents $i$ and $j$. Of course, if it is impossible for agent $i$ to produce what $j$ produces, $Q_{i}\left(z_{j}\right)$ will be undefined, and we will regard the equity* condition as being vacuously satisfied for these two agents.)

Admittedly this definition is not entirely ethically satisfactory. Perverse cases arise when one agent is the sole producer of some good, since in that case no complaint against him could be justified. It is especially bad if this agent is the sole producer of some good that gives utility only to him! However, in cases where there is a reasonable amount of substitution possibilities between agents' labor, the definition has a certain appeal. It only allows you to complain about another agent's consumption if you are willing to match his contribution to the social product. Otherwise your complaint cannot be considered legitimate. Thus I may "envy" a doctor who only works one day a week doing brain surgery and yet has substantial consumption; but unless I am willing to put in enough labor time to match his production of services -- for example, 10 years of medical school required -- my complaint against him 
cannot count as legitimate in the sense of equity*:

This definition does happily provide us with an existence theorem for fair* allocations; for the analog of Theorem 2.1 goes through:

THEOREM 3.1. If the Independence Assumption is satisfied, then if $(x, 1-q)$ is a strongly efficient allocation, then there is some agent that envies* no one, and some agent that no one envies*.

and so the existence theorem works:

THEOREM 3.2. Let X be regular, let preferences te monotonic, and suppose that there are no two allocations in PW which all agents regard as indifferent. Then fair* allocations exist.

(Of course the analog of Theorem 2.6 concerning the existence of fair sets goes through also.)

Recall that in the case of fair division, a competitive equilibrium from an equal division was fair. An analogous result holds for fair* allocations. Suppose that we have a particularly simple kind of technology where there we can associate with each agent an "ability", $a_{i}$, so that if the $i^{\text {th }}$ agent works for $q_{i}$ hours, he contributes $a_{i} q_{i}$ "labor power." Production then depends only on the amount of "labor power", not on the amount of time worked by agents. In this special case, an allocation is equitable* iff $\left(x_{i}, 1-q_{i}\right) \succ_{i}\left(x_{j}, 1-\left(a_{j} / a_{i}\right) q_{j}\right)$ for all $i$ and $j$. Then: 
THEOREM 3.3. Suppose that preferences are convex and monotonic; then if we choose an initial endowment where each agent gets $w / n$ of the consumption goods and 1 unit of his own leisure, the resulting competitive equilibrium is fair*.

So the "natural" equilibrium, with equal division and no compensation for abilities has the property of equity*: if any agent preferred some other agent's bundle to his own, he would not be willing to produce what that other agent produces.

We will now discuss the second concept of "fair allocations" that I mentioned earlier. If we have the classical conditions of convexity, monotonicity and so one, every efficient allocation is a competitive equilibrium for some initial endowment. Thus with each efficient allocation $(x, 1-q)$ we can associate a competitive price vector $(p, r)$ where $p$ is the price vector of the consumption goods and $r$ is the vector of wage rates for the various kinds of labor. We can then associate with each agent an implicit income $y_{i}=(p, r) \cdot\left(x_{i}\right.$, $1-q_{i}$ ) where each agent's leisure is evaluated at his particular wage rate. We will then say that an allocation is income-fair iff $y_{i}=y_{j}$ for all agents $i$ and $j$.

It is easy to prove that income-fair allocations will always exist; we simply divide the total consumptionleisure bundle up evenly by giving each agent an equal 
share of all consumption goods and an equal share of each other agent's labor time and then trade to a competitive equilibrium. Stated formally:

THEOREM 3.4. Suppose that preferences are convex and monotonic; then if we choose an initial endowment where each agent gets $\mathrm{w} / \mathrm{n}$ of the consumption goods and $1 / n$ of each agent's leisure, the resulting competitive equilibrium is income fair. ${ }^{2}$

Theorems 3.3 and 3.4 demonstrate the fundamental ambiguity of equity in the production case: should we view labor time on an individual basis and give each agent the same amount of his individual leisure, or should we view labor time on a social basis and give each agent the same amount of "labor power"? In the first case we have equal wealth but no correction for ability, and in the second case we have equal incomes and total correction for ability. 


\section{APPENDIX TO SECTION 3}

Note: For notation and definitions, refer to the Appendix to Section 2. In this section we will always assume that $w>0$, and that $x$ has a non-empty interior.

THEOREM 3.1. If the Independence Assumption is satisfied, then if $(x, 1-q)$ is a strongly efficient allocation, then there is some agent that envies* no one, and some agent that no one envies*.

Proof. The proof is similar to the proof of Theorem 2.1. Suppose that each agent envies* some other agent. Since there are only a finite number of agents there must be some cycle. Performing the "swap" among the agents in the cycle is feasible because of the definition of envy* and the Independence Assumption; the resulting allocation dominates the original one, contradicting efficiency.

THEOREM 3.2. Let $X$ be regular, let preferences be monotonic, and suppose that there are no two allocations in $\mathrm{PW}$ which all agents regard as indifferent. Then fair* allocations exist.

Proof. The proof is similar to that of Theorem 2.5 and Theorem 2.6. The first hypotheses allow the proof of Theorem 2.4 to work in this case, making the efficient 
set homeomorphic to a simplex. Since Theorem 4.1 provides the analog to Theorem 2.1, the application of the Knaster-Kuratowski-Mazurkiewicz lemma can procede as before.

THEOREM 4.3. Suppose that preferences are convex and monotonic; then if we choose an initial endowment where each agent gets $w / n$ of the consumption goods and 1 unit of his own leisure, the resulting competitive equilibrium is fair*.

Proof. The assumptions imply the existence of a competitive equilibrium with prices $p$ and wages $r$. We can normalize the wage of labor with ability 1 to have $r_{i}=$ 1 ; since in the competitive equilibrium al1 wages will be proportional to ability in this case, the normalization will make $r_{i}=a_{i}$ for $i=1, \ldots, n$. By the definition of competitive equilibrium we have

$$
\left(p, a_{i}\right) \cdot\left(x_{i}, 1-q_{i}\right)=p \cdot w / n+a_{i}
$$

or, rewriting,

$$
\mathrm{p} \cdot \mathrm{x}_{i}-\mathrm{a}_{\mathrm{i}} \mathrm{q}_{\mathrm{i}}=\mathrm{p} \cdot \mathrm{w} / \mathrm{n} \text { for } \mathrm{i}=1, \ldots, \mathrm{n} \text {. }
$$

It is clear that $(x, 1-q)$ is efficient; assume then that some agent $i$ envies* some agent $j$. Then

$$
\left(x_{i}, 1-q_{i}\right) \prec_{i}\left(x_{j}, 1-\left(a_{j} / a_{i}\right) q_{j}\right)
$$

which implies

$$
\left(p, a_{i}\right) \cdot\left(x_{i}, 1-q_{i}\right)<\left(p, a_{i}\right) \cdot\left(x_{j}, 1-\left(a_{j} / a_{i}\right) q_{j}\right)
$$


expanding and substituting we get

$$
\begin{gathered}
p \cdot x_{i}-a_{i} q_{i}<p \cdot x_{j}-a_{j} q_{j} \\
p \cdot w / n<p \cdot w / n
\end{gathered}
$$

which gives us the contradiction. $\square$ 
Example 3.1. The following economy ${ }^{1}$ has no fair allocations:

$$
\begin{aligned}
u_{1}\left(x_{1}, q_{1}\right)= & (11 / 10) x_{1}+\left(1-q_{1}\right) \\
u_{2}\left(x_{2}, q_{2}\right)= & 2 x_{2}+\left(1-q_{2}\right) \\
x_{1}+x_{2}= & q_{1}+(1 / 10) q_{2} \\
& 0 \leqq q_{1} \leqq 1 \\
& 0 \leqq q_{2} \leqq 1
\end{aligned}
$$

Proof.

(1) In any efficient allocation we must have:

(i) $q_{1}=1$ since if $q_{1}$ were strictly less than 1 we would have $u_{1}\left(x_{1}+\left(1-q_{1}\right), 0\right)$ strictly greater than $u_{1}\left(x_{1}, q_{1}\right)$ and that bundle would be feasible for agent 1 ;

(ii) $q_{2}=0$ since if $q_{2}$ were strictly greater than 0 we would have $u_{2}\left(x_{2}-e / 10, q_{2}-e\right)$ strictly greater than $u_{2}\left(x_{2}, q_{2}\right)$ and that bundle would be feasible for agent 2 , for small enough e.

(2) If $x_{2}=0$ we would have

$$
u_{2}\left(x_{1}, q_{1}\right)=2>1=u_{2}\left(x_{2}, q_{2}\right)
$$

which is certainly not fair.

(3) We are left with the case where the allocation is of the form $\left(\left(x_{1}, 1\right),\left(x_{2}, 0\right)\right)$ with $x_{1}+x_{2}=1$. For an allocation of this form to be fair we must have

$$
\begin{aligned}
& u_{1}\left(x_{1}, q_{1}\right) \geqq u_{1}\left(x_{2}, q_{2}\right) \\
& (11 / 10) x_{1} \geqq(11 / 10) x_{2}+1
\end{aligned}
$$

and 


$$
\begin{aligned}
& u_{2}\left(x_{2}, q_{2}\right) \geqq u_{2}\left(x_{1}, q_{1}\right) \\
& 2 x_{2}+1 \geqq 2 x_{1}
\end{aligned}
$$

But these two inequalities, along with the equality $x_{1}+x_{2}=1$ imply that $3 / 4 \geqq x_{1} \geqq 21 / 22$ which is a contradiction. $\square$ 


\section{NOTES AND ACKNOWLEDGEMENTS}

1. This example is due to Pazner and Schmeidler [PS1].

2. Pazner and Schmeidler prove a similar result in [PS2].

\section{REFERENCES}

[PS1] Pazner, E., and Schmeidler, D., "A Difficulty in the Concept of Equity," Department of Economics, Te1-Aviv University, November, 1972.

[PS2] Pazner, E., and Schmeidler, D., "Decentralization, Income Distribution, and the Role of Money in Socialist Economies," Technical Report No. 8, The Foerder Institute for Economic Research, Tel-Aviv University, September, 1972 . 


\section{COALITION FAIRNESS}

The concept of equity allows comparisons between agents to be made only on an individualistic basis: each agent compares his own bundle to the bundle of each of the other agents. A stronger notion of equity might be one in which comparisons were allowed between groups of agents. For example, each group of agents could compare its aggregate bundle to the aggregate bundle of any other group of the same size. A concept of this type will be called coalition fairness, or, more briefly, c-fairness. Before we can state the formal definitions, we will need to set up some machinery.

We consider a collection of agents, $C$, which may be finite or infinite, and the set of coalitions of agents in $C, C$, which we will assume to be a sigma-algebra of subsets of $C$. We have a measure on $C, \lambda: C \rightarrow \mathrm{R}_{+}$which measures the size of a coalition. If $C$ is finite, $\lambda$ will just be the normalized counting measure, while if $C$ is a continuum we will assume that $\lambda$ is an atomless measure, normalized so that $\lambda(C)=1$.

Suppose that we have some fixed bundle of goods, $\mathrm{W}$ in $\mathrm{R}_{+}^{\mathrm{k}}$, to be divided among the agents of $\mathrm{C}$. An allocation $\alpha$ will be a measure, $\alpha: C \rightarrow \mathrm{R}_{+}^{\mathrm{k}}, \alpha(\mathrm{C})=\mathrm{w}$, that assigns to each coalition its aggregate bundle. (In the finite case it is sometimes convenient to use the standard definition of allocation as an nk vector $x=\left(x_{1}, \ldots x_{n}\right)$.) Coalitions are assumed to have a preference ordering 
over possible allocations; we write "coalition A prefers $\alpha$ to $\beta$ " as " $\alpha \gg_{A} \beta$." Preferences are interpreted as " $\alpha \gg_{A} \beta$ " means "(almost) all agents in A prefer a to $B . "$ (See Debreu, [D].)

Finally, we denote by $\mathrm{R}_{\mathrm{e}}(\alpha)$ the e-range of $\alpha: \mathrm{R}_{\mathrm{e}}(\alpha)=$ $\{\alpha(A): \lambda(A)=e\}$, and by $P_{e}(\alpha)$ the e-preferred bundles, $\mathrm{P}_{\mathrm{e}}(\alpha)=\left\{\beta(\mathrm{A}): \beta \gg_{\mathrm{A}} \alpha\right.$ and $\left.\lambda(\mathrm{A})=\mathrm{e}\right\} \cdot \mathrm{R}_{\mathrm{e}}(\alpha)$ consists of all aggregate bundles held by coalitions of size e in the allocation $\alpha$, and $\mathrm{P}_{\mathrm{e}}(\alpha)$ consists of all aggregate bundles that can be distributed among the agents of a coalition of size e to form a preferred (partial) allocation.

We can now succinctly state the definition of c-fairness:

Definition. An allocation $\alpha$ is c-fair iff $\mathrm{P}_{\mathrm{e}}(\alpha) \cap \mathrm{R}_{\mathrm{d}}(\alpha)=$ $\emptyset$ for $0 \leqq \mathrm{~d} \leqq 1,0<\mathrm{e} \leqq 1$.

In other words, an allocation $\alpha$ is c-fair iff no coalition of size e prefers any aggregate bundle of any coalition of the same size or smaller. ${ }^{1}$

Notice that this definition requires that a c-fair allocation be (weakly) pareto efficient since for $e=\lambda(C)=$ 1 , we require that $P_{1}(\alpha) \cap R_{1}(\alpha)=\emptyset$, so that there is no way to rearrange the allocation to make every agent better off.

The first question is of course: when do c-fair allocations exist? Since the concept of c-fairness includes the concept of fairness, every c-fair allocation 
must certainly be fair but not vice versa. However, we do have:

THEOREM 4.1. If $\alpha$ is a competitive equilibrium with initial endowment $i(A)=\lambda(A) w$ for al1 $\mathrm{A}$ in $C$, then a is c-fair. ${ }^{2}$

In other words, equal income competitive equilibria are c-fair.

Are there any other c-fair allocations? In general the answer is "yes"; it is easy to construct examples in the two person, two good, Edgeworth box case. However, in an important sense, equal income competitive equilibria are the only c-fair allocations for a fair division problem with many agents.

There are two approaches to formalizing and demonstrating this proposition: one is by considering a replicated economy in the manner of Debreu and Scarf [DS]; the other is by considering an economy with an atomless continuum of agents in the manner of Vind [V1, V2]. We begin with the replicated economy.

Suppose that we have only a finite number of types of agents $i=1, \ldots, m$. Here, by saying that two agents are of the same type, I only mean that they have the same preferences. We will assume that these preferences are strongly convex, continuous, and insatiable, as do Debreu and Scarf [DS]. Under these assumptions, it is clear that c-fair allocations must have the equal treatment property; that is, if $\alpha$ is c-fair, then agents of the same type must 
get the same bundle. (This is proved formally in Lemma 4.0.)

Now consider a given allocation $x^{\prime}=\left(x_{1} \ldots x_{m}\right)$ which is c-fair, and let the economy replicate; that is, consider an economy with $r$ agents of each type and $r$ times the original bundle $w$ to be divided among them. (Admittedly this is not quite the fair division problem since the bundle to be divided keeps increasing as the economy replicates; however, since the number of agents keeps growing also, the problem is essentially the same from the viewpoint of an individual agent.)

Since c-fair allocations have the equal treatment property, we only need to consider their projection into the m type space, so clearly we will get no more of them in the replicated economy; the question is, will we get fewer? The answer is "yes"; in fact, we have:

THEOREM 4.2. If $\left(x_{1} \ldots x_{m}\right)$ is c-fair for all replications $\underline{r}$, then it is a competitive equilibrium with equal incomes; that is, with initial endowment $\omega_{i}=w / m$ for $i=1, \ldots, m$.

In the two person, two good, Edgeworth box case there is a simple diagrammatic argument which is presented as example 4.1 .

If c-fair allocations are equal income competitive allocations in the limit, we would expect that to be the case when we start out with a continuum of agents. As usual, we can also dispense with the assumption of convexity of preferences in the continuum case. 
First we note the following:

THEOREM 4.3. If $(C, C, \lambda)$ is an atomless economy and $\alpha$ is ac-fair allocation, then $\alpha$ is atomless. ${ }^{3}$

Thus we only need to consider atomless allocations as candidates for c-fair allocations. We now have:

THEOREM 4.4. If $(C, C, \lambda)$ is an atomless economy, then $\alpha$ is c-fair implies that $a$ is a competitive equilibrium with $i(A)=\lambda(A) w$ for a11 A in $C .^{4}$

The implication of Theorem 4.2 and Theorem 4.4 seems to be that if we wish to divide things fairly among a large number of agents so that the allocation is stable with respect to envy among coalitions, then our only choice is an allocation that is a competitive equilibrium with equal incomes.

It has been suggested to me that a more general and more symmetric definition of coalition fairness might be one where each coalition compares its "average" bundle to the "average" bundle of each other coalition. 5 In this way, each coalition can consider the aggregate bundles of al1 other coalitions, not just coalitions of the same size or smaller. We can formalize this notion in the following definition:

Definition. An allocation $\alpha$ is $c^{\prime}$-fair iff $\mathrm{P}_{\mathrm{e}}(\alpha) \cap(\mathrm{e} / \mathrm{d})$ $\mathrm{R}_{\mathrm{d}}(\alpha)=\emptyset$ for $a 110<\mathrm{d} \leqq 1$ and $0<\mathrm{e} \leqq 1$.

This each coalition examines the aggregate bundle of each other coalition, weighting the aggregate bundle by 
their relative sizes; if any such weighted bundle is preferred by the examining coalition, the allocation cannot be $c^{\prime}$-fair.

It is clear that the notion of $c^{\prime}$-fairness implies the notion of c-fairness, and one would suspect that it is a strictly stronger notion; that is, that there are c-fair allocations that are not c'-fair. However, that is not the case:

THEOREM 4.5. Let $(C, C, \lambda)$ be an atomless economy; then an allocation $\alpha$ is $c^{\prime}$-fair if and only if it is c-fair. 


\section{APPENDIX TO SECTION 4}

Definitions :

An allocation $\alpha$ is in the (equal division) core iff there is no allocation $\beta$ and coalition $B$ such that $B \succcurlyeq_{B} \alpha$ and $\beta(B)=\lambda(B) w$ for $\lambda(B)>0$.

An allocation $\alpha$ is a competitive equilibrium from $i(A)=\lambda(A) w$ iff there exists a price vector $p$ in $R^{k}$ such that $p \cdot \alpha(A)=p \cdot i(A)$ for all $A$ in $C$ and $p \cdot x>p \cdot i(A)$ for a $11 \mathrm{x}$ in $\mathrm{P}_{\mathrm{e}}(\alpha)$, A in $C$ such that $\lambda(\mathrm{A})=\mathrm{e}>0$.

A coalition $A$ is an atom for a measure $\mu$ iff $\mu(A)>0$ and $B \subset A$ implies that $\mu(B)=\mu(A)$ or $\mu(B)=0$.

Assumptions on preferences:

(1) In the replication case we make the assumptions of Debreu and Scarf [DS]; namely,

(i) Insatiability. Given a commodity bundle $x$ we assume that there is a commodity bundle $x^{\prime}$ such that $x^{\prime} \succ_{i} x$

(ii) Strong-convexity. Let $x^{\prime}$ and $x$ be arbitrary commodity bundles, $x^{\prime} \neq x, x^{\prime} \succ_{i} x$, and let $0<a<1$. We assume that $a x+(1-a) x^{\prime} \succ_{i} x$.

(iii) Continuity. We assume that $\left\{x: x \succsim_{i} x^{\prime}\right\}$ and $\left\{x: x \delta_{i} x^{\prime}\right\}$ are closed.

(2) In the continuum case we make the assumptions of Vind [V1]; namely, 
(i) Independence. The preferences of a coalition A are independent of the values of the allocation outside of A.

(ii) Monotonicity. If an allocation $\alpha$ gives at least the same amount as $\beta$ to all subcoalitions of $A$ and more of at least one commodity to all subcoalitions of $\mathrm{A}$ with $\beta(B) \neq 0$, then $A$ prefers $\alpha$ to $\beta$ and to any allocation worse than $\beta$.

(iii) Continuity. If $\beta$ is preferred to $\alpha$ by $A$, then any commodity bunde in a neighborhood of $B(A)$ can be allocated to $A$ in such a way that the new allocation is still preferred to $\alpha$ by A.

Facts:

THEOREM. (Debreu and Scarf) If the assumptions of (1) above are met, and if $\left(x_{1}, \ldots, x_{m}\right)$ is in the (equal division) core for all replications $r$, then it is a competitive equilibrium with equal incomes.

THEOREM. (Vind) If the assumptions of (2) above are met, then for $(C, C, \lambda)$ an atomless economy, the (equal division) core exactly coincides with the set of allocations that are competitive equilibria from $i(A)=\lambda(A) w$ for $A$ in $C$.

THEOREM. (Lyaponov) The range of a vector valued measure is closed and convex. 
LEMMA 4.0. If preferences are strongly convex, then c-fair allocations have the equal treatment property; that is, if $x$ is a c-fair allocation and the preferences of agent $i$ are exactly the same as the preferences of agent $j$, then $x_{i}=x_{j}$.

Proof. Suppose that the total number of agents is $\mathrm{r}_{\mathrm{n}}$, and let $\mathrm{e}=2 / \mathrm{n}$. Setting $z=x_{i}+x_{j}$ we have that $z$ is in $\mathrm{R}_{\mathrm{e}}(\alpha)$, for $\alpha$ the measure corresponding to the allocation $x$.

Since $x$ is c-fair, $x_{i} \sim_{i} x_{j}$ and $x_{j} \infty_{j} x_{i} \cdot$ Assuming that $x_{i} \neq x_{j}$ we have that $y=(1 / 2) x_{i}+(1 / 2) x_{j}$ is preferred by both $i$ and $j$ to their present bundles by strong convexity, and thus the aggregate bundle $2 y=z$ is in $P_{e}(\alpha)$. Since $P_{e}(\alpha)$ and $R_{e}(\alpha)$ are thus not disjoint, the allocation $x$ cannot be c-fair. This is a contradiction.

THEOREM 4.1. If $\alpha$ is a competitive equilibrium with initial endowment $i(A)=\lambda(A) w$ for all A in $C$, then $\alpha$ is c-fair. ${ }^{2}$

Proof. Assume not; then there is some coalition A that prefers to have the bundle of some coalition $B$ with $\lambda(\mathrm{B}) \leqq \lambda(\mathrm{A})$. Thus, $\mathrm{p} \cdot \alpha(\mathrm{B})>\mathrm{p} \cdot \alpha(\mathrm{A})$. But $\mathrm{i}(\mathrm{A})=$ $\lambda(A) w \geqq \lambda(B) w=i(B)$ so that $p \cdot i(B) \leqq p \cdot i(A)$, contradicting the definition of a competitive equilibrium. 
THEOREM 4.2. If $\left(x_{1}, \ldots, x_{m}\right)$ is c-fair for all replications $\underline{r}$, then it is a competitive equilibrium with equal incomes; that is, with initial endowment $\omega_{i}=w / m$ for $i=1, \ldots, m$. Proof. Suppose that $x=\left(x_{1}, \ldots, x_{m}\right)$ is c-fair but is not in the (equal division) $r$-core for some replication $r$. Then we wish to show that eventually $x$ cannot be c-fair. If $x$ is not in the $r$-core, then it is blocked by some coalition $S$ that has $s$ members, $N_{i}(S)$ members of each type, $i=1, \ldots, m$. Let $z$ be the (partial) allocation that is preferred by $S$ that is feasible for it; we have

$$
i=1 \sum_{q=1}^{m} z_{i q}^{r}=s w / m
$$

(Here $z_{i q}$ refers to the bundle of an agent of type $i$ in replication $q$, for agent iq in $S_{\text {.) }}$

Let MS be the coalition consisting of $\mathrm{mN}_{i}(S)$ agents of type $i=1, \ldots, m$. This is just an m-times replication of $\mathrm{S}$ and thus has ms members. Furthermore, it blocks $\left(x_{1}, \ldots, x_{m}\right)$ via the (partial) allocation $z^{\prime}$ where $z$ ' is just the m-times replication of $z$ and thus

$$
i=1 \sum_{q=1}^{m} z_{i q}^{r m}=s m w / m=s w
$$

Consider now the coalition SM consisting of $s$ copies of each of the original m types of agents. SM is the same size as MS; furthermore, at the original allocation $x$, SM holds $s \sum_{i=1}^{m} x_{i}=s w$ so that the (partial) allocation $z^{\prime}$ is feasible for it and the allocation $z^{\prime}$ is preferred by MS to its 
allocation, showing that $x$ is not c-fair.

Therefore, if $x$ is c-fair for every replication $r$, it must eventually be in every $\mathrm{r}$-core, and thus it must be a competitive equilibrium with equal incomes, by the theorem of Debreu and Scarf. $\square$

THEOREM 4.3. If $(C, C, \lambda)$ is an atomless economy and $\alpha$ is a c-fair allocation, then a is atomless. ${ }^{3}$

Proof. Assume not and let $A$ be an atom for $\alpha$; by definition of atom $\alpha(\mathrm{A})>0$. There are two cases:

(1) $\lambda(A)=0$. Since there can be at most only a countable number of atoms of $\alpha$, we can find a non-atomic coalition $B$ with $\lambda(B)>\lambda(A)$ and $\alpha(B)<\alpha(A)$. This contradicts the assumption that $\alpha$ is c-fair.

(2) $0<\lambda(\mathrm{A}) \leqq 1$. Let $\mathrm{A}=\mathrm{B} \cup \mathrm{D}$ with $\lambda(\mathrm{B})=\lambda(\mathrm{D})=$ $(1 / 2) \lambda(A)$. Then $\alpha(B)+\alpha(D)=\alpha(A)$ and yet either

$$
\begin{aligned}
& \text { (i) } \alpha(B)=0 \text { and } \alpha(D)=\alpha(A) \text {, or } \\
& \text { (ii) } \alpha(D)=0 \text { and } \alpha(B)=\alpha(A) .
\end{aligned}
$$

In either case, $\alpha$ is not c-fair.

THEOREM 4.4. If $(\mathrm{C}, C, \lambda)$ is an atomless economy, then $\alpha$ is c-fair implies that $\alpha$ is a competitive equilibrium with $i(A)=\lambda(A) w$ for all A in $C$.

Proof. Assume that $\alpha$ is not a competitive equilibrium with $i(A)=\lambda(A) w$ for all $\mathrm{A}$ in $C$. Then $\alpha$ is not in the (equal division) core. Thus there are $(\beta, B)$ such that $\beta \gg_{B} \alpha$ 
and $B(B)=i(B)=\lambda(B) w$, so $\lambda(B) w$ is in $P_{e}(\alpha)$ for $e=\lambda(B)$.

Consider now the range of the vector valued measure $(\alpha, \lambda)$. Certainly $(\alpha(C), 1)$ is in it and certainly $(0,0)$ is in it; by convexity $(\lambda(B) \alpha(C), \lambda(B))$ is in it.. Since $\alpha(C)=w$, this implies that $\lambda(B) w$ is in $R_{e}(\alpha)$ for $e=\lambda(B)$, which contradicts the fact that $\alpha$ is c-fair.

THEOREM 4.5. Let $(C, C, \lambda)$ be an atomless economy; then an allocation $\alpha$ is $c^{\prime}$-fair if and only if it is c-fair.

Proof. Sufficiency. This is equivalent to " $\alpha$ is not c-fair" implies that " $\alpha$ is not c'-fair." If $\alpha$ is not c-fair, then for some d less than or equal to some e, $\mathrm{P}_{\mathrm{e}}(\alpha) \cap \mathrm{R}_{\mathrm{d}}(\alpha) \neq \emptyset$ so that there is some bundle $\mathrm{x}$ in the intersection. Then $(e / d) x \geq x$ so that $(e / d) x$ is in $\mathrm{P}_{\mathrm{e}}(\alpha)$ by monotonicity implying that $\alpha$ is not $c^{\prime}-\mathrm{fair}$. Necessity. If $\alpha$ is c-fair, it is a competitive equilibrium with prices $\mathrm{p}$ such that $\mathrm{p} \cdot \alpha(\mathrm{A})=\mathrm{p} \cdot \lambda(\mathrm{A}) \mathrm{w}$ for all $\mathrm{A}$ in $C$, by Theorem 4.4. Suppose that $\alpha$ is not $c^{\prime}$-fair; then $(e / d) \alpha(B)$ is in $P_{e}(\alpha)$ for some $B$ with $\lambda(B)=d$. By definition of competitive equilibrium, we have $\mathrm{p} \cdot(\mathrm{e} / \mathrm{d}) \alpha(\mathrm{B})>\mathrm{p} \cdot \mathrm{we}$. But $\mathrm{p} \cdot \alpha(B)=\mathrm{p} \cdot \lambda(B) \mathrm{w}$ so that $\mathrm{p} \cdot \lambda(\mathrm{B}) \mathrm{w}(\mathrm{e} / \mathrm{d})>\mathrm{p} \cdot \mathrm{we}$, which implies that $\mathrm{p} \cdot \mathrm{we}>\mathrm{p} \cdot \mathrm{we}$ since $\lambda(B)=d$. This is a contradiction. $\square$ 
Example 4.1. Consider the example depicted below where $x$ is c-fair, since it is preferred by both agents to its swap $x^{\prime}$, but $x$ is not a competitive equilibrium with equal

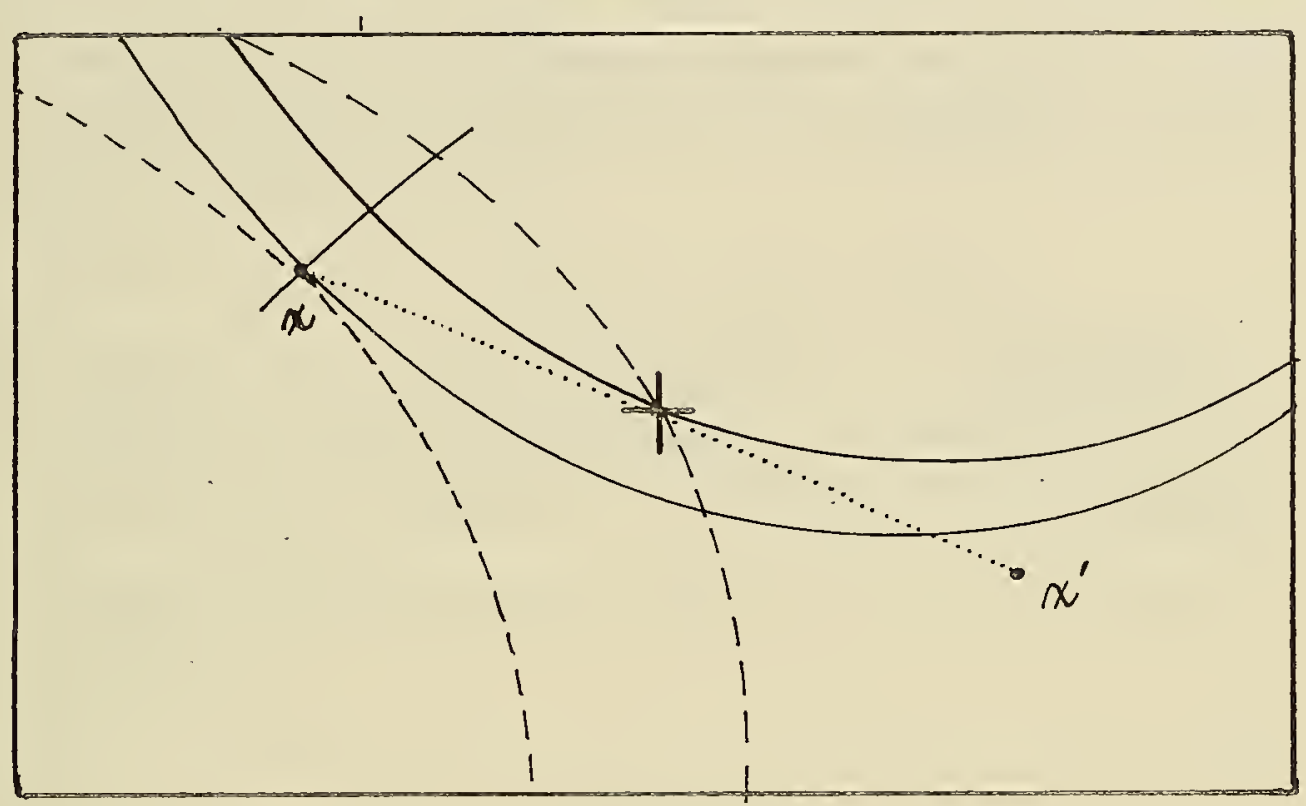

A

incomes or even in the (equal division) 1-core.

Let the economy replicate once so that we have two agents of each type and consider the coalition AA consisting of two agents of type $A$ and the coalition $A B$ consisting of one agent of type $A$ and one agent of type $B$.

Since $x$ is outside of the core, both agents of type A prefer the allocation $w / 2$ to what they hold at $x$. But the allocation $w / 2$ is feasible for the coalition $A B$ and thus AA prefers the aggregate bundle of AB so $x$ is not c-fair.

A similar argument can be made for any allocation that is outside of the $r$-core for any replication $r$. Since the core shrinks down to the competitive equilibrium this is the only allocation that can be c-fair for all r. 
NOTES AND ACKNOWLEDGEMENTS

1. The definition of c-fairness is due to Vind [V2].

2. Theorem 4.1 was stated and proved by Vind in [V2]. A quite general theorem on the existence of a competitive equilibrium with a continuum of agents may be found in [A].

3. Theorem 4.3 was stated by Vind in [V2].

4. Theorem 4.4 is a generalization of a theorem by Vind in [V2] which had required the additional hypothesis that the dimension of $\left(U R_{e}(\alpha)\right)=k$. Since this hypothesis is economically meaningless the present version is a substantial improvement.

5. Andreu Mas-Collel and Michael Scriven made this suggestion.

\section{REFERENCES}

[A] Auman, R., "Existence of Competitive Equilibria in Markets with a Continuum of Traders," Econometrica, Vol. 34, No. 1, (January, 1966)

[D] Debreu, G., "Preference Functions on Measure Spaces of Economic Agents," Econometrica, Vol. 35, No. 1, (January, 1967)

[DS] Debreu, G., and Scarf, H., "A Limit Theorem on the Core of an Economy," International Economic Review, Vol. 4, No. 3, (September, 1963)

[V1] Vind, K., "Edgeworth-Allocations in an Exchange Economy with Many Traders," International Economic Review, Vo1. 5, No. 2, (May, 1964)

[V2] Vind, K., "Lecture Notes for Economics 288 ," Stanford University, Spring, 1971, (xeroxed) 





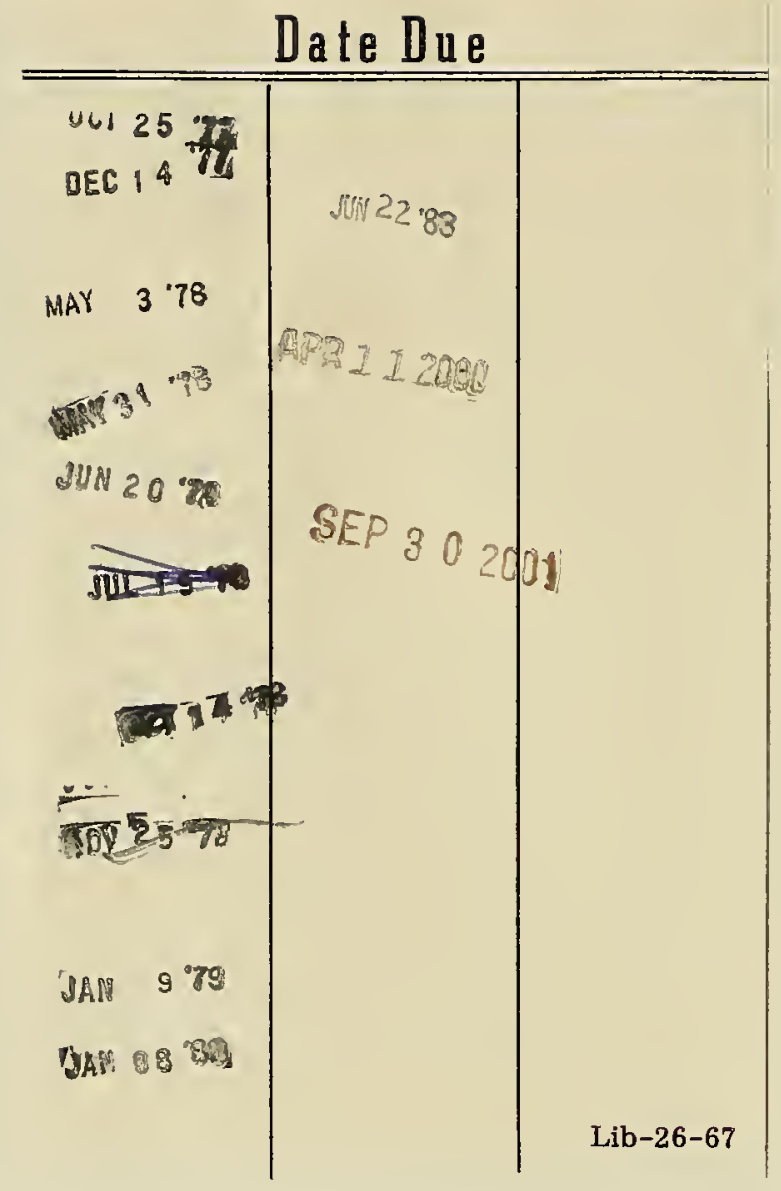




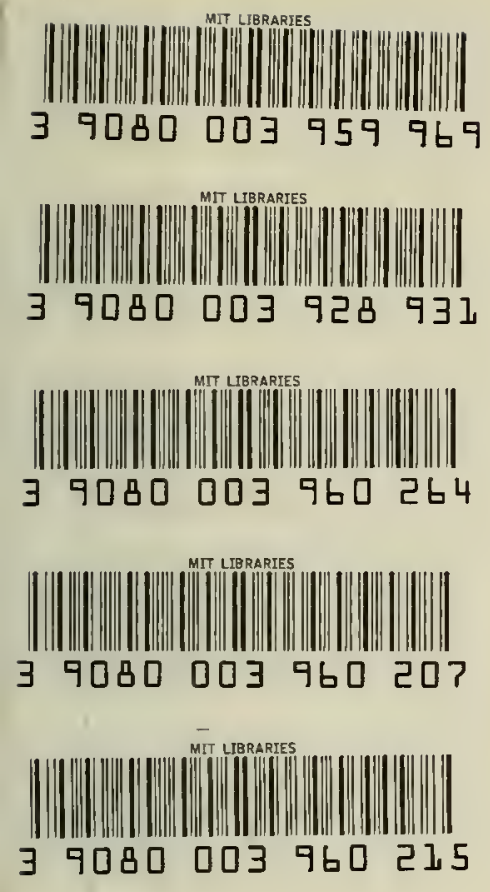


